\title{
Convex Hodge Decomposition of Image Flows *
}

\author{
Jing Yuan ${ }^{1}$, Gabriele Steidl ${ }^{2}$, Christoph Schnörr ${ }^{1}$ \\ 1 Image and Pattern Analysis Group, Heidelberg Collaboratory for Image Processing, \\ University of Heidelberg, Germany, \{yuanjing, schnoerr\} @math.uni-heidelberg.de \\ 2 Faculty of Mathematics and Computer Science, University of Mannheim, Germany, \\ steidl@math.uni-mannheim.de
}

\begin{abstract}
The total variation (TV) measure is a key concept in the field of variational image analysis. Introduced by Rudin, Osher and Fatemi in connection with image denoising, it also provides the basis for convex structure-texture decompositions of image signals, image inpainting, and for globally optimal binary image segmentation by convex functional minimization. Concerning vector-valued image data, the usual definition of the TV measure extends the scalar case in terms of the $L^{1}$-norm of the gradients.

In this paper, we show for the case of $2 \mathrm{D}$ image flows that TV regularization of the basic flow components (divergence, curl) leads to a mathematically more natural extension. This regularization provides a convex decomposition of motion into a richer structure component and texture. The structure component comprises piecewise harmonic fields rather than piecewise constant ones. Numerical examples illustrate this fact. Additionally, for the class of piecewise harmonic flows, our regularizer provides a measure for motion boundaries of image flows, as does the TV-measure for contours of scalar-valued piecewise constant images.
\end{abstract}

\section{Introduction}

The total variation (TV) measure has been introduced by Rudin, Osher and Fatemi [1] in connection with image denoising. The precise definition will be given in Section 2. In the following, let $\Omega \subset \mathbb{R}^{2}$ be a bounded domain. Minimizing the convex functional

$$
\frac{1}{2}\|u-d\|_{\Omega}^{2}+\lambda \operatorname{TV}(u)
$$

for given image data $d$ leads to an edge-preserving nonlinear smoothing process that effectively removes noise and small-scale spatial patterns from $d$.

Starting with the work of Meyer [2], the more general viewpoint of image decomposition has been adopted - see [3] and references therein. The basic model is again given by (1), leading to a decomposition

$$
d=u+v
$$

\footnotetext{
* This work was supported by the German Science Foundation (DFG), grant Schn $457 / 9-1$
} 
of given image data $d$ in a piecewise constant image structure $u$ and oscillatory patterns and noise $v$.

Another key property of the TV measure is due to its geometric interpretation via the co-area formula [4]: TV $(u)$ equals the length of level lines of $u$, summed up over the range of $u$ (contrast). As a consequence, $\operatorname{TV}(u)$ measures the length of contours of piecewise constant images, hence implements the regularization term of the Mumford-Shah variational approach to segmentation. This fact has been explored for image inpainting [5] and more recently also for image segmentation, in order to replace non-convex variational approaches $[6,7]$ by convex ones that can be globally optimized $[8,9]$.

A natural issue concerns the application of the TV measure to vector-valued data $u=\left(u_{1}, u_{2}\right)^{\top}$. Usually definitions are applied that, for sufficiently regular $u$ (cf. next section), take the form [10]

$$
\operatorname{TV}(u)=\int_{\Omega} \sqrt{\left|\nabla u_{1}\right|^{2}+\left|\nabla u_{2}\right|^{2}} d x .
$$

The Helmholtz decomposition of $u$ into its basic components, divergence and curl, suggest an alternative:

$$
\int_{\Omega} \sqrt{(\operatorname{div} u)^{2}+(\operatorname{curl} u)^{2}} d x .
$$

This viewpoint has been suggested recently in [11] for decomposing image flows. However, a geometric interpretation and its connection to the definitions (3) and (1) has not been given. It will turn out below, that

- (4) is a mathematically more natural definition extending (1), and that

- (4) decomposes flows into a richer "structural" component - analogous to $u$ in the scalar case (2) - comprising piecewise harmonic flows rather than piecewise constant flows.

The paper is organized as follows. We recall necessary material of TV-based scalar image decomposition in Section 2. Next, we consider the Helmholtz and Hodge decompositions of vector fields into orthogonal subspaces in Section 3. Comparing by analogy the latter decomposition to the scalar-valued case, and then re-considering the convex TV-based decomposition of scalar images in Section 2, we are led in Section 4 to the interpretation of (4) as a generalized convex decomposition of image flows. Section 5 embedds this decomposition into a variational denoising approach for image flows. We illustrate this result and its consequences numerically in Section 6 and conclude in Section 7.

\section{TV-Based Convex Image Decomposition}

The total variation of a scalar function $u$ is defined as

$$
\operatorname{TV}(u)=\sup _{\|p\|_{\infty} \leq 1}\langle u, \operatorname{div} p\rangle_{\Omega},\left.\quad p\right|_{\partial \Omega}=0,
$$


where $\|p\|_{\infty}=\max _{x \in \Omega} \sqrt{p_{1}^{2}(x)+p_{2}^{2}(x)}$ and $p \in C_{0}^{1}\left(\Omega ; \mathbb{R}^{2}\right)$. Based on this definition, the convex variational image denoising approach (1) can be transformed into its dual formulation [12]

$$
\min _{\|p\|_{\infty} \leq 1}\left\{\|\lambda \operatorname{div} p-d\|_{\Omega}^{2}\right\}, \quad d=u+\lambda \operatorname{div} p
$$

This results in a decomposition of scalar image data $d$,

$$
d=u+\lambda \operatorname{div} p, \quad\|p\|_{\infty} \leq 1,\left.\quad p\right|_{\partial \Omega}=0
$$

where $u$ provides the piecewise smooth component, i.e. the large-scale structural parts of the image signal $d$, and $\lambda \operatorname{div} p$ comprises the corresponding small-scale structure, i.e. texture and noise. To this end, it is also called the structure-texture decomposition.

In order to be consistent with the following definitions, we rewrite the TV-based scalar decomposition as

$$
d=u+\operatorname{div} p, \quad\|p\|_{\infty} \leq \lambda,\left.\quad p\right|_{\partial \Omega}=0 .
$$

The small-scale component $\operatorname{div} p$ is the orthogonal projection

$$
\Pi: L^{2}(\Omega) \rightarrow \operatorname{div} C_{\lambda}
$$

of the data $d$ onto a convex set $\operatorname{div} C_{\lambda}$, i.e. onto the image of the set of normconstrained vector fields

$$
C_{\lambda}=\left\{\left(p_{1}, p_{2}\right)^{\top} \mid\|p\|_{\infty} \leq \lambda\right\}
$$

under the divergence operator. For $d$ with mean zero, the smallest value of $\lambda$ such that the solution $u$ of (1) becomes zero equals the G-norm of $d$ (cf. [2]) which measures the size of the small-scale oscillating component (noise, texture) of the image function.

Hence, we formally write

$$
L^{2}(\Omega)=U \oplus_{\Pi} \operatorname{div} C_{\lambda},
$$

where the index of $\oplus_{\Pi}$ indicates that this is a convex decomposition, rather than an orthogonal decomposition into subspaces.

\section{Orthogonal Hodge Decomposition of Image Flows}

In this section, we summarize orthogonal decomposition of vector fields, the Helmholtz decomposition and, as an extension thereof, the Hodge decomposition. The latter decomposition together with (9) will provide the basis for our novel decomposition of image flows in the subsequent section. 
Theorem 1 (2D Orthogonal Decomposition). Any vector field $u \in\left(L^{2}(\Omega)\right)^{2}$ can be decomposed into into an irrotational and a solenoidal part

$$
u=v+\nabla^{\perp} \phi, \quad \phi_{\partial \Omega}=0,
$$

where $\operatorname{curl} v=0$. This decomposition is unique, and the components are orthogonal in the sense $\left\langle v, \nabla^{\perp} \phi\right\rangle_{\Omega}=0$.

The representation (10) corresponds to an orthogonal decomposition of the space of all vector fields into subspaces of gradients and curls

$$
\left(L^{2}(\Omega)\right)^{2}=\nabla H(\Omega) \oplus \nabla^{\perp} H_{o}(\Omega),
$$

where $H(\Omega)$ and $H_{o}(\Omega)$ are suitably defined Sobolev-type spaces. We refer to [13] for details.

The curl-free component can be further decomposed into

$$
v=h+\nabla \psi,
$$

where $\psi_{\partial \Omega}=0$ and

$$
\operatorname{div} v=\operatorname{div} \nabla \psi
$$

The component $h$ satisfies

$$
\operatorname{div} h=0, \quad \operatorname{curl} h=0,
$$

and is called harmonic flow. We summarize these facts:

Theorem 2 (2D Hodge Decomposition). Let $\Omega$ be a bounded, sufficiently regular $2 D$ domain (Poincaré lemma [13]). Then any vector field $u \in\left(L^{2}(\Omega)\right)^{2}$ can be decomposed into

$$
u=h+\nabla \psi+\nabla^{\perp} \phi, \quad \phi_{\partial \Omega}=0, \psi_{\partial \Omega}=0,
$$

where $\operatorname{div} h=\operatorname{curl} h=0$. This decomposition is unique, and the three parts on the right-hand side are orthogonal to each other.

Accordingly, the decomposition (11) can be refined to

$$
\left(L^{2}(\Omega)\right)^{2}=\mathcal{H} \oplus \nabla H_{o}(\Omega) \oplus \nabla^{\perp} H_{o}(\Omega),
$$

where the additional index of the second component indicates the boundary condition $\psi_{\partial \Omega}=0$, and where $\mathcal{H}$ denotes the space of all harmonic flows.

\section{Convex Hodge Decomposition of Image Flows}

In this section, we first provide an orthogonal decomposition of scalar-valued functions analogously to the orthogonal decomposition of vector fields in the previous section. Next, we generalize this to a novel convex decomposition of image flows, in view of the convex decomposition of scalar functions described in Section 2. 


\subsection{Orthogonal Decomposition of Scalar-Valued Functions}

We look for an orthogonal decomposition of scalar-valued image functions, analogous to the decomposition (13) for vector fields.

Any scalar-valued function $d \in L^{2}(\Omega)$ can be decomposed into

$$
d=c+\operatorname{div} p,\left.\quad p\right|_{\partial \Omega}=0 .
$$

Since $\int_{\Omega} \operatorname{div} p d x=0$, due to the boundary condition on $p$, the constant function $c$ is just the mean value $\int_{\Omega} d d x /|\Omega|$, and we have also the orthogonality of the two components $\langle c, \operatorname{div} p\rangle_{\Omega}=0$. Referring to (13), it makes sense to regard (15) as Hodge decomposition of scalar fields.

Next, let us consider the connection between (15) and the convex decomposition (7). As in (7), $d$ is decomposed into two components, a smooth component and a remaining component of small-scale structure.

The difference between (7) and (15) is that the latter projects onto special

convex sets: orthogonal subspaces.

This leads to a constant "smooth" component $c$ in (15), as opposed to $u$ in (7) that tends to be piecewise constant. Of course, if $\lambda$ is large enough, then $u$ in (7) also becomes a constant.

Likewise, analogous to the convex decomposition (9), we obtain as a result of (15) the orthogonal decomposition

$$
L^{2}(\Omega)=H_{c} \oplus \operatorname{div} P,
$$

where $H_{c}$ is the set of all constant functions on $\Omega$, and $P$ the subspace of all vector fields vanishing on the boundary $\partial \Omega$.

\subsection{Convex Decomposition of Vector Fields}

Based on the previous discussion, natural questions arise:

1. Because the orthogonal decomposition of scalar fields (15), (16) is clearly inferior to the convex decomposition (7), (9) in that the structural component $u$ models more general functions, what is the convex counterpart of the orthogonal decomposition of vector fields (13), (14)?

2. What is the natural generalization of the variational denoising approach (1) to the case of vector fields?

We will address the first question next and the second question in the following section.

Definition 1 (Convex Hodge Decomposition). Given a vector field $d \in$ $\left(L^{2}(\Omega)\right)^{2}$, we define the convex decomposition

$$
d=u+\nabla \psi+\nabla^{\perp} \phi,
$$


where the non-smooth component $u$ is given by the orthogonal projection of $d$ onto the convex set

$$
S_{\lambda}=\left\{\nabla \psi+\nabla^{\perp} \phi \mid(\psi, \phi) \in C_{\lambda} \cap\left(H_{o}(\Omega)\right)^{2}\right\},
$$

with $C_{\lambda}$ from (8). Formally, write

$$
\left(L^{2}(\Omega)\right)^{2}=U_{\mathcal{H}} \oplus_{\Pi}\left\langle\left(\nabla, \nabla^{\perp}\right), C_{\lambda}\right\rangle,
$$

where $\Pi$ is the orthogonal projector onto $S_{\lambda}(18)$.

Note the structural similarity between (7), (9) and (17), (19). Likewise, as $U$ in (9) generalizes $H_{c}$ in (16) from constant functions to piecewise constant functions, so $U_{\mathcal{H}}$ in (19) generalizes $\mathcal{H}$ in (14) to piecewise harmonic vector fields.

\section{Variational Denoising of Image Flows}

In this section, we investigate a natural generalization of the variational approach (1) to vector fields. First, we present the objective function and reveal its connection to the convex decomposition (17). Next, we show that for the particular case of piecewise harmonic vector fields, it regularizes motion boundaries in a similar way as the functional (1) does for the contours of piecewise constant and scalar-valued functions.

\subsection{Variational Approach}

For a given vector field $d$, we study the convex functional

$$
\inf _{u}\left\{\frac{1}{2}\|u-d\|_{\Omega}^{2}+\lambda R(u)\right\}
$$

with

$$
R(u)=\sup _{s \in S}\langle s, u\rangle_{\Omega},
$$

where $S=S_{1}$ from (18). Because $R(u)$ is positively homogeneous, $\lambda R(u)$ equals $\sup _{s \in S_{\lambda}}\langle s, u\rangle_{\Omega}$. Hence, (20) reads

$$
\inf _{u} \sup _{s \in S_{\lambda}}\left\{\frac{1}{2}\|u-d\|_{\Omega}^{2}+\langle s, u\rangle_{\Omega}\right\}
$$

Exchanging inf and sup yields the convex decomposition (17) (cf. (18))

$$
d=u+s,
$$

and as the dual convex problem of (1) the projection of the data $d$ onto the convex set $S_{\lambda}$

$$
\inf _{s \in S_{\lambda}}\|s-d\|_{\Omega}^{2}, \quad d=u+\nabla \psi+\nabla^{\perp} \phi
$$


Let us point out again the similarities of our approach for denoising vector fields with the ROF-model (1) for denoising scalar-valued functions, based on the convex Hodge decomposition introduced in the previous section. The regularizer $R(u)$ in (21) is defined as support function of a convex set, as is the total variation measure TV $(u)$ in (5). Likewise, the dual convex problem (22) characterizes the non-smooth, oscillating component ("motion texture") of a given vector field $d$ as a projection onto a convex set, as does the ROF-model for scalar-valued functions in (6).

\subsection{Motion Segmentation and Piecewise Harmonic Image Flows}

We take a closer look to the analogy between the ROF-model and our generalization to vector fields, as discussed in the previous section.

Assuming $u$ to be sufficiently smooth, and taking into account the representation (18) and that the boundary values of functions $\psi, \phi$ representing an element $s \in S$ vanish, we rewrite the regularizing term (21)

$$
\begin{aligned}
R(u) & =\sup _{s \in S}\langle s, u\rangle_{\Omega}=\sup _{\|(\psi, \phi)\|_{\infty} \leq 1}\left\langle\nabla \psi+\nabla^{\perp} \phi, u\right\rangle_{\Omega} \\
& =\sup _{\|(\psi, \phi)\|_{\infty} \leq 1}\left\{-\langle\psi, \operatorname{div} u\rangle_{\Omega}-\langle\phi, \operatorname{curl} u\rangle_{\Omega}\right\} \\
& =\int_{\Omega} \sqrt{(\operatorname{div} u)^{2}+(\operatorname{curl} u)^{2}} d x .
\end{aligned}
$$

This differs from the commonly used term (3). In particular, for a piecewise harmonic flow (i.e. with almost everywhere vanishing divergence and curl), corresponding to the structural part of the decomposition (19), this measure only contributes at motion boundaries. In fact, we can show that $R(u)$ plays a similar role as does the TV-measure at discontinuities of scalar-valued functions [14].

\section{$6 \quad$ Numerical Examples}

The main contribution of this paper is the mathematically thorough investigation of non-smooth convex regularization of image flows, based on the decomposition framework presented in Section 4, and leading to the variational approach (20) in Section 5 that naturally extends the ROF-model (1) to the vector-valued case.

In this section, we confine ourselves to underline the difference between the novel approach (20) and the commonly used regularization term (3) with few numerical experiments.

Figure 1 shows an image flow additively composed of a harmonic flow and a second small-scale motion component. While the usual regularization (3) always returns a constant vector field as structural part, the regularizer (21) is able to separate these two components. This result clearly validates the theory.

Figure 2 presents a more involved non-rigid flow composed of several components, a global harmonic flow and four very local constant ones (i.e. harmonic as well), and further two local non-harmonic local flows. Figures 3 and 4 show the 

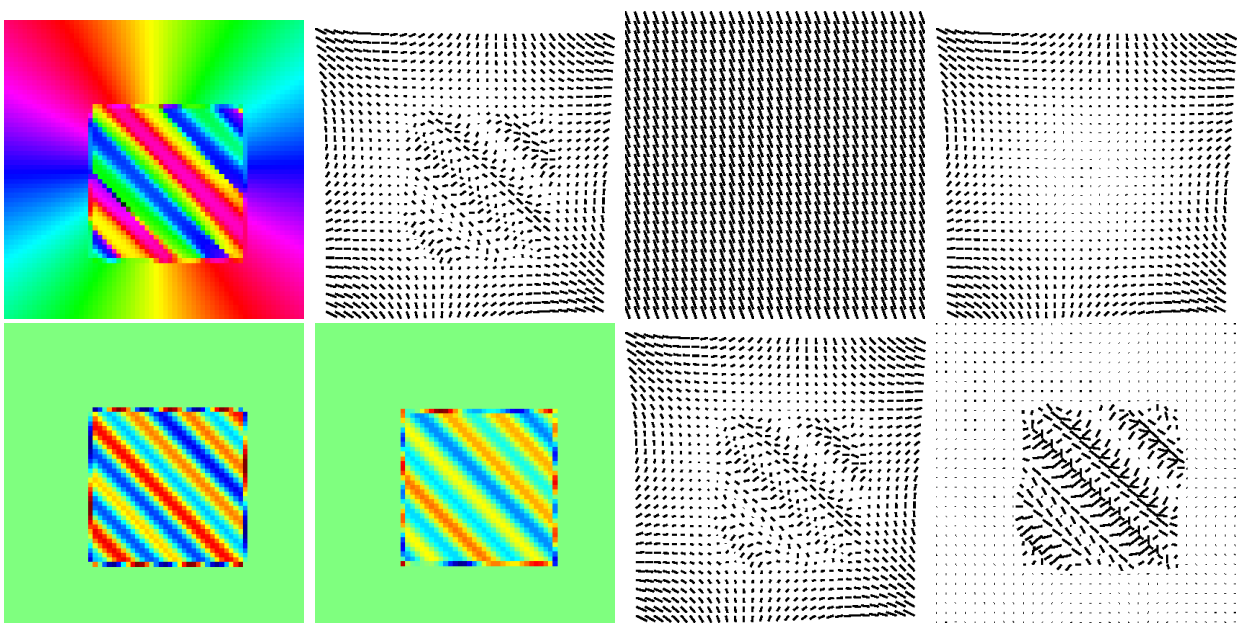

Fig. 1. Left two columns: A flow comprising a harmonic component and motion texture as vector plot and orientation plot (top) and its divergence and curl (bottom). Third column: Standard TV-regularization is unable to separate the flows. Right column: Our novel regularizer clearly separates motion texture and nonrigid flows.

results for the usual TV method (3) und our approach for two different values of the regularization parameter $\lambda$ that determines what large-scale and small-scale motion structures mean. The key observation is that the (3) does not lead to a meaningful result, whereas the novel term (23) clearly separates motion discontinuities and non-harmonic flows from harmonic ones, depending on their scale relative to the value of $\lambda$.

\section{Conclusions}

Our work enlarges the class of convex variational approaches that can be used to denoise and separate - in our case - vector-valued data. Our results elucidate a further mathematical setting where to some extent decisions (separation and segmentation) can be done just by convex programming, that is globally optimal.

Our further work will explore the connection of spaces of harmonic flows to other low-dimensional spaces of flows that are relevant for applications.

Acknowledgement This work has been supported by the German Science Foundation (DFG), grant Schn 457/9-1.

\section{References}

1. L. Rudin, S. Osher, and E. Fatemi. Nonlinear total variation based noise removal algorithms. Physica D, 60:259-268, 1992. 


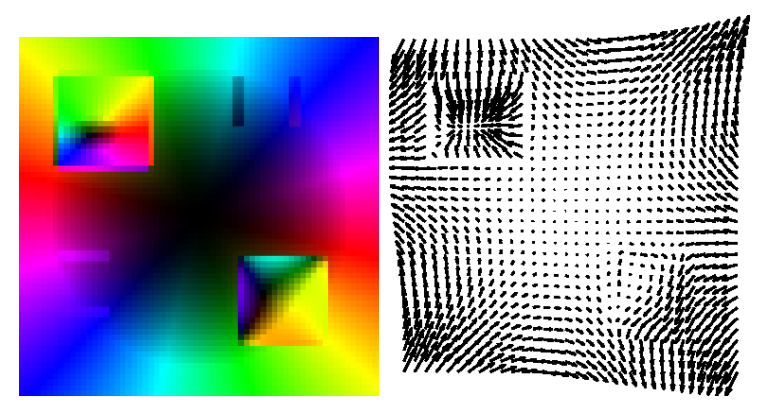

Fig. 2. A complex flow - orientation plot (left) and vector plot (right) - comprising a single global harmonic flow, four very local constant flows, and two further local nonharmonic flows. The addition of all components provides the input data for convex variational denoising.
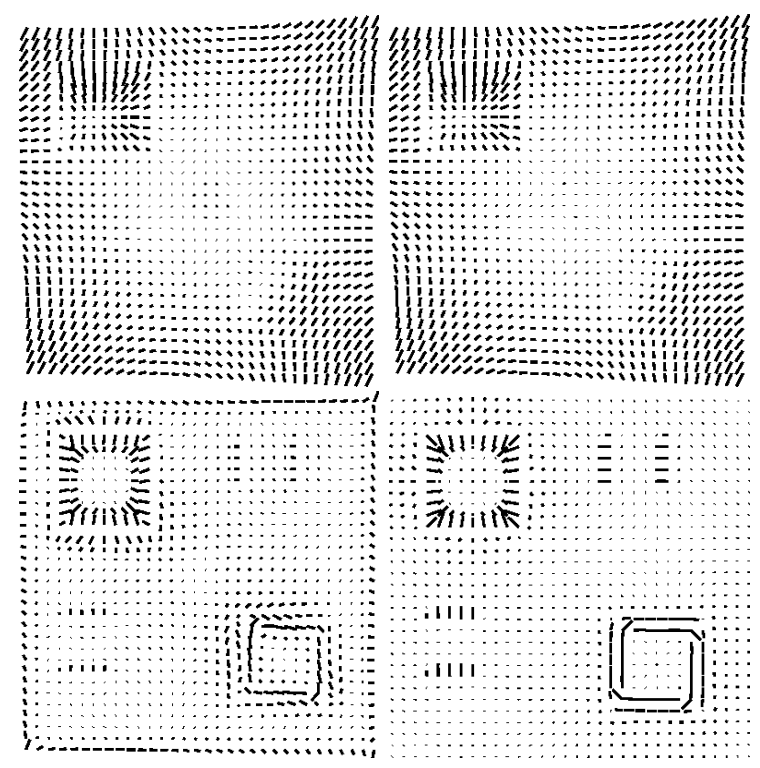

Fig. 3. Left column: Standard TV regularization always mixes up the flow components of Figure 2. Right column: Our novel geometric regularization term always captures - depending on the scale parameter $\lambda$ - motion discontinuities and non-harmonic flow components.

2. Y. Meyer. Oscillating Patterns in Image Processing and Nonlinear Evolution Equations, volume 22 of Univ. Lect. Series. Amer. Math. Soc., 2001.

3. J.-F. Aujol, G. Gilboa, T. Chan, and S. Osher. Structure-texture image decomposition - modeling, algorithms, and parameter selection. Int. J. Comp. Vision, 67(1):111-136, 2006.

4. W.P. Ziemer. Weakly Differentiable Functions. Springer, 1989.

5. T.F. Chan and J. Shen. Mathematical models for local non-texture inpaintings. SIAM J. Appl. Math., 61(4):1019-1043, 2001. 


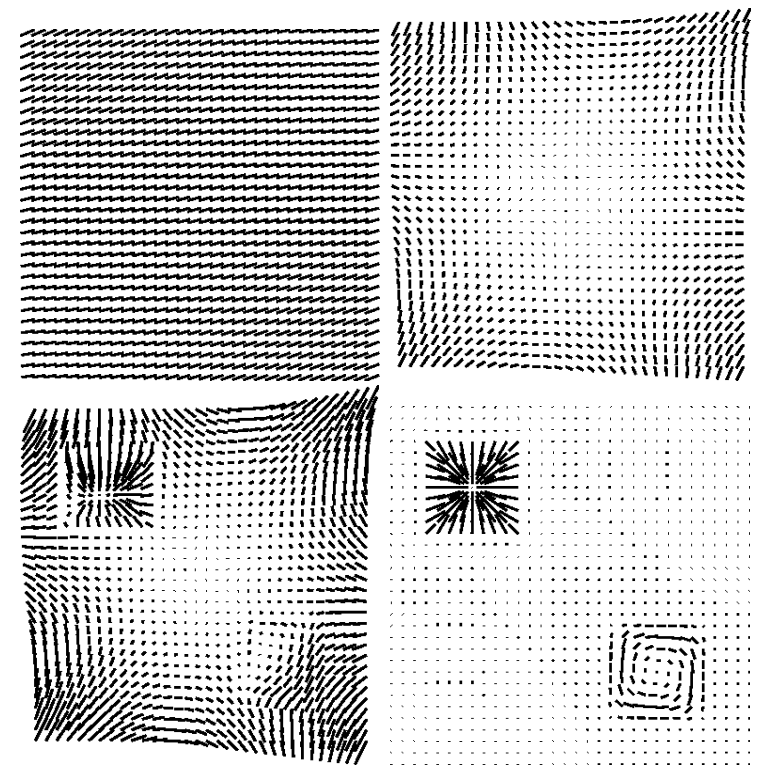

Fig. 4. Same results as shown in Figure 3, but for a larger value of the scale parameter $\lambda$. Left column: Independent of this different $\lambda$-value, standard TV-regularization just filters out a constant flow. Right column: Due to the larger value of the scale parameter $\lambda$, only the smooth global harmonic flow is returned as structural part by the novel regularizer (top, right), whereas all smaller-scale flows are regarded and separated as motion texture (bottom, right).

6. D. Mumford and J. Shah. Optimal approximations by piecewise smooth functions and associated variational problems. Comm. Pure Appl. Math., 42:577-685, 1989.

7. T.F. Chan and L.A. Vese. Active contours without edges. IEEE Trans. Image Proc., 10(2):266-277, 2001.

8. T.F. Chan, S. Esedoglu, and M. Nikolova. Algorithms for finding global minimizers of image segmentation and denoising models. SIAM J. Appl. Math., 66(5):1632$1648,2006$.

9. X. Bresson, S. Esedoglu, P. Vandergheynst, J.-P. Thiran, and S. Osher. Fast global minimization of the active contour/snake model. J. Math. Imag. Vision, 28(2):151$167,2007$.

10. T.F. Chan and J. Shen. Image Processing and Analysis. Cambridge Univ. Press, 2005.

11. J. Yuan, C. Schnörr, and G. Steidl. Simultaneous optical flow estimation and decomposition. SIAM J. Scientific Computing, 29(6):2283-2304, 2007.

12. A. Chambolle. An algorithm for total variation minimization and applications. J. Math. Imag. Vision, 20:89-97, 2004.

13. V. Girault and P.-A. Raviart. Finite Element Methods for Navier-Stokes Equations. Springer, 1986.

14. J. Yuan, G. Steidl, and C. Schnörr. Convex hodge decompositions of image flows. Submitted. 